\title{
Velocity and stress fields in grounded glaciers: a simple algorithm for including deviatoric stress gradients
}

\author{
HeINZ BLATTER \\ Geographisches Instilut, Eidgenössische Technische Hochschule, CH-8057 Zürich, Switzerland
}

\begin{abstract}
A new and efficient algorithm for computing the three-dimensional stress and velocity fields in grounded glaciers includes the role of deviatoric stress gradients. A consistent approximation of first order in the aspect of ratio of the ice mass gives a set of eight field equations for the five stress and three velocity components and the corresponding boundary conditions. A coordinate transformation mapping the local ice thickness on to unity and approximating the derivatives in the horizontal direction by centred finite-differences yields five ordinary differential and three algebraic equations. This allows use of the method of lines, starting the integration with prescribed stress and velocity components at the base, and a simple iteration procedure converges rapidly.

The algorithm can be used for a wide range of stress strain-rate relations, as long as strain only depends on deviatoric and shear stresses and on temperature. Sensitivity tests using synthetic and realistic ice geometries show the relevance of normal deviatoric stresses in the solutions for the velocity components even for ice sheets. Stress and velocity fields may deviate substantially from the widely used shallow-ice approximation.
\end{abstract}

\section{INTRODUCTION}

This paper explores a new and efficient algorithm for computing the three-dimensional stress and velocity fields in glaciers and ice sheets including the role of normal deviatoric stress gradients. The algorithm is based on a two-dimensional version developed by Muller (1991). A set of field equations and boundary conditions in a consistent approximation of first order in the aspect ratio of the ice mass can be solved using the method of lines and a simple iteration procedure. The algorithm is effective for a wide range of stress-strain-rate relations (flow laws), as long as only deviatoric and shear stresses are involved. Temperature-dependence of the flow rate may be introduced through a temperature-dependent rate factor.

The mechanics and thermodynamics of an ice mass constitute a Stokes problem, where the geometry and the temperature field evolve with time, but the velocity and stress fields are quasi-stationary. This allows one to calculate the flow as a steady-state field for a given transient geometry and transient viscosity field at a given time. In this work, the evolution of the geometry and of the temperature field are not considered. The viscosity field is, of course, the result of transient evolution of other internal fields, such as the temperature, moisture content, impurities and crystal size.

It is generally assumed that deviatoric stress gradients are negligibly small for the modelling of the overall behaviour of large ice sheets, although it is admitted that this may not be true near ice divides and near ice margins. However, attempts to model ice-sheet evolution over several glacial-interglacial cycles demand a fast algorithm. This consideration forces more restrictive approximations (Mahaffy, 1976; Herterich, 1988; Huybrechts, 1992), such as the shallow-ice approximation rigorously established by Hutter (1983). On the other hand, Dahl-Jensen (1989) showed that longitudinal deviatoric stress and shear stress are both of lead order for a plane-strain transverse section of the Greenland ice sheet and neither one can be neglected. The same holds even more for glaciers with larger aspect ratios than ice sheets, where deviatoric stress gradients account for important features, such as crevassing and wavy-surface topography.

Obviously, there is no single algorithm optimal for each application and numerical methods must be chosen according to the type of study. The advantage of the numerical scheme promoted in this study is its simplicity and easy application for an interesting range of ice-sheet and glacier situations. To demonstrate this, the algorithm is applied to a simple synthetic ice-sheet geometry and is also used to simulate infinitely long parallel-sided slabs. A scaling tailored to the type of geometry reduces the number of parameters and thus allows the performance of the algorithm to be investigated for a wide range of sizes and aspect ratios.

\section{GOVERNING EQUATIONS}

\subsection{Field equations}

Ice is treated as an incompressible viscous fluid. Its mechanical properties may depend on other fields of 
physical quantities, such as temperature and stress. The geometry of the ice mass is defined by the upper free surface $S=S(x, y)$ and the basal surface $B=B(x, y)$ in Cartesian coordinates $(x, y, z)$ with the $z$ axis pointing opposite to the direction of gravity. The equation for mass continuity then becomes

$$
\frac{\partial u}{\partial x}+\frac{\partial v}{\partial y}+\frac{\partial w}{\partial z}=0
$$

where $u, v$ and $w$ are the velocity components in the $x, y$ and $z$ directions, respectively, and for linear momentum

$$
\begin{aligned}
& \frac{\partial \tau_{x x}}{\partial x}+\frac{\partial \tau_{x y}}{\partial y}+\frac{\partial \tau_{x z}}{\partial z}=0 \\
& \frac{\partial \tau_{x y}}{\partial x}+\frac{\partial \tau_{y y}}{\partial y}+\frac{\partial \tau_{y z}}{\partial z}=0 \\
& \frac{\partial \tau_{x z}}{\partial x}+\frac{\partial \tau_{y z}}{\partial y}+\frac{\partial \tau_{z z}}{\partial z}=\rho g
\end{aligned}
$$

where $\rho$ is the density of ice, $g$ is the acceleration of gravity and $\tau_{x x}, \tau_{y y}, \tau_{z z}, \tau_{x z}, \tau_{y z}$ and $\tau_{x y}$ are the components of the symmetric Cauchy stress tensor $\tau_{i j}$. Glacier ice is generally treated as a non-Newtonian fluid with a stress-strain-rate relation of the form

$$
\begin{aligned}
\frac{\partial u}{\partial x} & =A F(\cdot) \sigma_{x x}, \\
\frac{\partial v}{\partial y} & =A F(\cdot) \sigma_{y y}, \\
\frac{1}{2}\left(\frac{\partial u}{\partial z}+\frac{\partial w}{\partial x}\right) & =A F(.) \tau_{x z}, \\
\frac{1}{2}\left(\frac{\partial v}{\partial z}+\frac{\partial w}{\partial y}\right) & =A F(.) \tau_{y z}, \\
\frac{1}{2}\left(\frac{\partial u}{\partial y}+\frac{\partial v}{\partial x}\right) & =A F(.) \tau_{x y}
\end{aligned}
$$

where $\sigma_{i j}=\tau_{i j}-\left(\frac{1}{3}\right) \tau_{k k} \delta_{i j}$ is the deviatoric stress tensor and $\sigma_{x x}$ and $\sigma_{y y}$ are the normal deviatoric stress components. Due to the incompressiblity condition in Equation (2.1), an additional equation relating $\partial w / \partial z$ to $\sigma_{z z}$ is redundant and can be omitted. The term $A F(\cdot)$ represents a flow law. In this study, a flow law of the form (Nye, 1953; Glen, 1958; Meier, 1958)

$$
A F(\cdot)=A\left(T^{2}+T_{0}^{2}\right)^{(n-1) / 2}=\frac{1}{2 \mu}
$$

is used, where $T$ is the effective stress (second invariant of the deviatoric stress tensor), $\mu$ is the viscosity and $\mu_{0}=1 / A T_{0}{ }^{n-1}$ is the viscosity in the limit of vanishing stress. The rate factor $A$ is usually assumed to depend exclusively on temperature. The absence of pressuredependence, e.g. considered in Weertman (1973), is crucial for the applicability of the algorithm introduced below. Substituting Equations (2.5) and (2.6) into Equation (2.1) yields

$$
\frac{\partial w}{\partial z}=-A F(\cdot)\left(\sigma_{x x}+\sigma_{y y}\right),
$$

which is easier to handle than Equation (2.1) in the numerical integration scheme described in sections 3.1 and 3.2 .

For the upper free surface $S=S(x, y)$, the boundary conditions for stress are

$$
\begin{aligned}
& n_{x} \tau_{x x}+n_{y} \tau_{x y}+n_{z} \tau_{x z}=-n_{x} P, \\
& n_{x} \tau_{x y}+n_{y} \tau_{y y}+n_{z} \tau_{y z}=-n_{y} P, \\
& n_{x} \tau_{x z}+n_{y} \tau_{y z}+n_{z} \tau_{z z}=-n_{z} P
\end{aligned}
$$

where

$$
n_{x}=\frac{\partial S}{\partial x}, \quad n_{y}=-\frac{\partial S}{\partial y} \quad \text { and } \quad n_{z}=1
$$

are the components of the normal vector $\mathbf{n}$ pointing outward from the ice and $P$ is the pressure on the outer side of the surface. Note, in this simple form, the vector $\mathbf{n}$ is not a unit vector. In the case of a grounded ice sheet with no basal melt or freezing and no isostatic uplift, basal motion is to a good approximation parallel to the bed

$$
w_{\mathrm{b}}=u_{\mathrm{b}} \frac{\partial B}{\partial x}+v_{\mathrm{b}} \frac{\partial B}{\partial y},
$$

in the case of sliding, and $u=v=w=0$ in the case of non-sliding conditions.

\subsection{Scale analysis}

To arrive at a consistent simplified set of equations, we need to estimate the order of magnitude of the various terms in the equations. To this end, we introduce a scaling for spatial variables $x, y, z, S$ and $B$,

$$
(x, y, z, S, B)=\{L\}(\tilde{x}, \tilde{y}, \epsilon \tilde{z}, \epsilon \tilde{S}, \epsilon \tilde{B}),
$$

the velocity components $u, v$ and $w$,

$$
(u, v, w)=A_{0}\{H\}(\rho g\{H\} \epsilon)^{n}(\tilde{u}, \tilde{v}, \epsilon \tilde{w}),
$$

the stress components $\tau_{i j}, \sigma_{k k}$ and the pressure $P$,

$$
\left(\tau_{i j}, \sigma_{k k}, P\right)=\rho g\{H\} \epsilon\left(\tilde{\tau}_{i j}, \tilde{\sigma}_{k k}, \tilde{P}\right),
$$

and for the rate factor $A$

$$
A=A_{0} \tilde{A}
$$

where $\{L\}$ and $\{H\}$ denote the characteristic horizontal and vertical extents of the ice sheet, respectively, $A_{0}$ is a typical rate factor, and $\tilde{x}, \tilde{y}, \tilde{z}, \tilde{S}, \tilde{B}, \tilde{u}, \tilde{v}, \tilde{w}, \tilde{\tau}_{i j}, \tilde{\sigma}_{i j}, \tilde{P}$ and $\tilde{A}$ are the corresponding dimensionless scaled variables of order unity. This specific scaling only applies if we assume ice behaves according to the generalized Glen flow law (Equation (2.10)). Since the extent of an ice sheet is much larger than its thickness, the aspect ratio $\epsilon=\{H\} /\{L\} \ll 1$ is used as scaling parameter. We define the first-order approximation by deleting terms of order $\epsilon^{2}$ but retaining terms of order 1 and $\epsilon$. Although the first-order approximation can also be considered as a shallow-ice approximation (Dahl-Jensen, 1989), the term "shallow-ice approximation" will be used only for the "zeroth"-order approximation in the rest of this paper; for simplicity, the first-order shallow-ice approximation will be referred to as "first-order" approximation.

Scaling the continuity Equation (2.11), the stress Equations (2.2)-(2.4) and the constitutive Equations 
$(2.5)-(2.9)$ yield

$$
\begin{gathered}
\epsilon \frac{\partial \tilde{w}}{\partial \tilde{z}}=-\tilde{A} \tilde{F}(\cdot)\left(\tilde{\sigma}_{x x}+\tilde{\sigma}_{y y}\right), \\
\epsilon \frac{\partial \tilde{\tau}_{x x}}{\partial \tilde{x}}+\epsilon \frac{\partial \tilde{\tau}_{x y}}{\partial \tilde{y}}+\frac{\partial \tilde{\tau}_{x z}}{\partial \tilde{z}}=0, \\
\epsilon \frac{\partial \tilde{\tau}_{x y}}{\partial \tilde{x}}+\epsilon \frac{\partial \tilde{\tau}_{y y}}{\partial \tilde{y}}+\frac{\partial \tilde{\tau}_{y z}}{\partial \tilde{z}}=0, \\
\epsilon^{2} \frac{\partial \tilde{\tau}_{x z}}{\partial \tilde{x}}+\epsilon^{2} \frac{\partial \tilde{\tau}_{y z}}{\partial \tilde{y}}+\epsilon \frac{\partial \tilde{\tau}_{z z}}{\partial \tilde{z}}=1, \\
\epsilon \frac{\partial \tilde{u}}{\partial \tilde{x}}=\tilde{A} \tilde{F}(\cdot) \tilde{\sigma}_{x x}, \\
\epsilon \frac{\partial \tilde{v}}{\partial \tilde{y}}=\tilde{A} \tilde{F}(\cdot) \tilde{\sigma}_{y y}, \\
\frac{\partial \tilde{u}}{\partial \tilde{z}}+\epsilon^{2} \frac{\partial \tilde{w}}{\partial \tilde{x}}=2 \tilde{A} \tilde{F}(\cdot) \tilde{\tau}_{x z}, \\
\frac{\partial \tilde{v}}{\partial \tilde{z}}+\epsilon^{2} \frac{\partial \tilde{w}}{\partial \tilde{y}}=2 \tilde{A} \tilde{F}(\cdot) \tilde{\tau}_{y z}, \\
\epsilon\left(\frac{\partial \tilde{u}}{\partial \tilde{y}}+\frac{\partial \tilde{v}}{\partial \tilde{x}}\right)=2 \tilde{A} \tilde{F}(\cdot) \tilde{\tau}_{x y}
\end{gathered}
$$

and the surface-boundary conditions for stress in Equations $(2.12)-(2.14)$ in the scaled form are

$$
\begin{gathered}
-\epsilon \frac{\partial \tilde{S}}{\partial \tilde{x}} \tilde{\tau}_{x x}-\epsilon \frac{\partial \tilde{S}}{\partial \tilde{y}} \tilde{\tau}_{x y}+\tilde{\tau}_{x z}=\epsilon \frac{\partial \tilde{S}}{\partial \tilde{x}} \tilde{P}_{\text {air }}, \\
-\epsilon \frac{\partial \tilde{S}}{\partial \tilde{x}} \tilde{\tau}_{x y}-\epsilon \frac{\partial \tilde{S}}{\partial \tilde{y}} \tilde{\tau}_{y y}+\tilde{\tau}_{y z}=\epsilon \frac{\partial \tilde{S}}{\partial \tilde{y}} \tilde{P}_{\text {air }}, \\
-\epsilon \frac{\partial \tilde{S}}{\partial \tilde{x}} \tilde{\tau}_{x z}-\epsilon \frac{\partial \tilde{S}}{\partial \tilde{y}} \tilde{\tau}_{y z}+\tilde{\tau}_{z z}=-\tilde{P}_{\text {air }} .
\end{gathered}
$$

To eliminate the normal Cauchy stresses in Equations $(2.22)-(2.24)$, we follow the usual procedure (see e.g. Herterich, 1987) by deleting terms of order $O\left(\epsilon^{2}\right)$ in Equation (2.24) and integrating the truncated equations from $\bar{z}$ to the surface $S$ and applying the result in Equations (2.22) and (2.23). Integration yields

$$
\tilde{\tau}_{z z}=\tilde{\tau}_{z z}(\tilde{S})-\frac{1}{\epsilon}(\tilde{S}-\tilde{z})
$$

Using Equation (2.32) and the relation between Cauchy stresses and deviatoric stresses in the form

$$
\tilde{\tau}_{z z}=\tilde{\tau}_{x x}-\tilde{\sigma}_{y y}-2 \tilde{\sigma}_{x x}=\tilde{\tau}_{y y}-\tilde{\sigma}_{x x}-2 \tilde{\sigma}_{y y}
$$

in Equation (2.33) and dropping terms of order $O\left(\epsilon^{2}\right)$ yields

$$
\begin{aligned}
& \tilde{\tau}_{x x}=\tilde{\sigma}_{y y}+2 \tilde{\sigma}_{x x}-\frac{1}{\epsilon}(\tilde{S}-\tilde{z})-\tilde{P}_{\text {air }}, \\
& \tilde{\tau}_{y y}=\tilde{\sigma}_{x x}+2 \tilde{\sigma}_{y y}-\frac{1}{\epsilon}(\tilde{S}-\tilde{z})-\tilde{P}_{\text {air }} .
\end{aligned}
$$

We differentiate Equations (2.35) and (2.36) and substitute the result in Equations (2.22) and (2.23), respectively, to obtain

$$
\begin{aligned}
& \epsilon\left(2 \frac{\partial \tilde{\sigma}_{x x}}{\partial \tilde{x}}+\frac{\partial \tilde{\sigma}_{y y}}{\partial \tilde{x}}+\frac{\partial \tilde{\tau}_{x y}}{\partial \tilde{y}}\right)+\frac{\partial \tilde{\tau}_{x z}}{\partial \tilde{z}}=\frac{\partial \tilde{S}}{\partial \tilde{x}}, \\
& \epsilon\left(2 \frac{\partial \tilde{\sigma}_{y y}}{\partial \tilde{y}}+\frac{\partial \tilde{\sigma}_{x x}}{\partial \tilde{y}}+\frac{\partial \tilde{\tau}_{x y}}{\partial \tilde{x}}\right)+\frac{\partial \tilde{\tau}_{y z}}{\partial \tilde{z}}=\frac{\partial \tilde{S}}{\partial \tilde{y}} .
\end{aligned}
$$

Similarily, eliminating $\tilde{\tau}_{x x}, \tilde{\tau}_{y y}$ and $\tilde{\tau}_{z z}$ in the boundary conditions in Equations (2.30)-(2.32) and dropping terms of order $\epsilon^{2}$ gives the corresponding first-order boundary conditions (Morland and Shoemaker, 1982; Morland, 1984)

$$
\begin{aligned}
& \tilde{\tau}_{x z}-\epsilon \frac{\partial \tilde{S}}{\partial \tilde{x}}\left(2 \tilde{\sigma}_{x x}-\tilde{\sigma}_{y y}\right)+\epsilon \frac{\partial \tilde{S}}{\partial \tilde{y}} \tilde{\tau}_{x y}=0, \\
& \tilde{\tau}_{y z}-\epsilon \frac{\partial \tilde{S}}{\partial \tilde{y}}\left(2 \tilde{\sigma}_{y y}-\tilde{\sigma}_{x x}\right)+\epsilon \frac{\partial \tilde{S}}{\partial \tilde{x}} \tilde{\tau}_{x y}=0 .
\end{aligned}
$$

Finally, we get eight independent Equations (2.37), (2.38), (2.21) and $(2.25)-(2.29)$ for the eight unknown field variables $\tilde{\sigma}_{x x}, \tilde{\sigma}_{y y}, \tilde{\tau}_{x z}, \tilde{\tau}_{x y}, \tilde{\tau}_{y z}, \tilde{u}, \tilde{v}$ and $\tilde{w}$. This set of eight equations only constitutes a well-posed problem for the eight unknown field variables, if the rate factor $\tilde{A}$ is independent of pressure (i.e. of normal Cauchy stresses).

\section{NUMERICAL SCHEME}

\subsection{Coordinate transformation}

The numerical scheme used for integrating the above set of first-order equations was developed by Muller (1991) for the two-dimensional plane-strain approximation, and is extended here for the three-dimensional case. We apply a coordinate transformation Phillips, 1957; Jenssen, 1977)

$$
\zeta=\frac{\tilde{z}-\tilde{B}(\tilde{x}, \tilde{y})}{\tilde{S}(\tilde{x}, \tilde{y})-\tilde{B}(\tilde{x}, \tilde{y})},
$$

which maps the local ice thickness on to unity (Fig. 1). Equations (2.37), (2.38), (2.21) and $(2.25)-(2.29)$ are transformed to

$$
\begin{aligned}
\frac{\partial \hat{\tau}_{x}}{\partial \zeta}= & -\epsilon\left[\frac{\partial c_{y}}{\partial \zeta} \tilde{\tau}_{x y}+\frac{\partial c_{x}}{\partial \zeta}\left(2 \tilde{\sigma}_{x x}+\tilde{\sigma}_{y y}\right)\right] \\
& -(\tilde{S}-\tilde{B})\left[\epsilon\left(2 \frac{\partial \tilde{\sigma}_{x x}}{\partial \tilde{x}}+\frac{\partial \tilde{\sigma}_{y y}}{\partial \tilde{x}}+\frac{\partial \tilde{\tau}_{x y}}{\partial \tilde{y}}\right)-\frac{\partial \tilde{S}}{\partial \tilde{x}}\right],
\end{aligned}
$$

$$
\begin{aligned}
\frac{\partial \hat{\tau}_{y}}{\partial \zeta}= & -\epsilon\left[\frac{\partial c_{x}}{\partial \zeta} \tilde{\tau}_{x y}+\frac{\partial c_{y}}{\partial \zeta}\left(2 \tilde{\sigma}_{y y}+\tilde{\sigma}_{x x}\right)\right] \\
& -(\tilde{S}-\tilde{B})\left[\epsilon\left(2 \frac{\partial \tilde{\sigma}_{y y}}{\partial \tilde{y}}+\frac{\partial \tilde{\sigma}_{x x}}{\partial \tilde{y}}+\frac{\partial \tilde{\tau}_{x y}}{\partial \tilde{x}}\right)-\frac{\partial \tilde{S}}{\partial \tilde{y}}\right],
\end{aligned}
$$




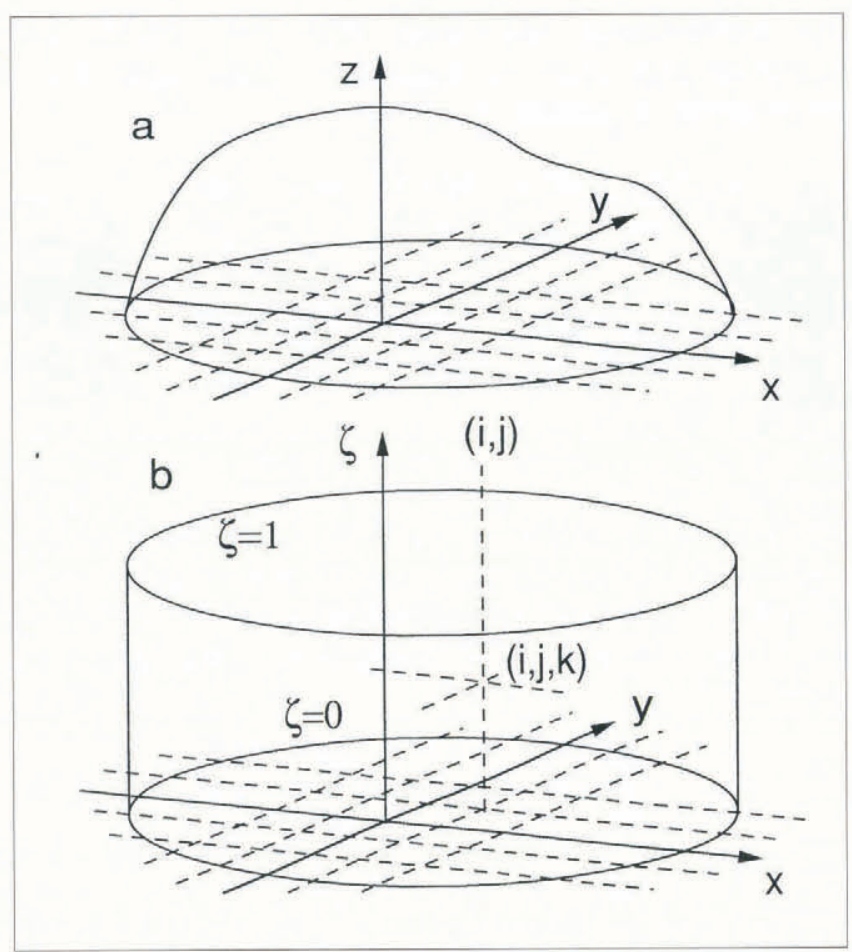

Fig. 1. Typical ice-sheet shape (a) and the transformed shape (b) by mapping the ice thickness on to unily. The grid is uniform in the transformed coordinates.

$$
\begin{gathered}
\frac{\partial \tilde{w}}{\partial \zeta}=-\frac{1}{\epsilon}(\tilde{S}-\tilde{B}) \tilde{A} \tilde{F}\left(\tilde{\sigma}_{x x}+\tilde{\sigma}_{y y}\right) \\
\frac{\partial \tilde{u}}{\partial \zeta}=2(\tilde{S}-\tilde{B}) \tilde{A} \tilde{F}\left[\hat{\tau}_{x}+\epsilon\left(c_{y} \tilde{\tau}_{x y}+c_{x} \tilde{\sigma}_{y y}+2 c_{x} \tilde{\sigma}_{x x}\right)\right]
\end{gathered}
$$

$$
\frac{\partial \tilde{v}}{\partial \zeta}=2(\tilde{S}-\tilde{B}) \tilde{A} \tilde{F}\left[\hat{\tau}_{y}+\epsilon\left(c_{x} \tilde{\tau}_{x y}+c_{y} \tilde{\sigma}_{x x}+2 c_{y} \tilde{\sigma}_{y y}\right)\right],
$$

$$
0=\epsilon \frac{\partial \tilde{u}}{\partial \tilde{x}}-\tilde{A} \tilde{F}\left(2 \epsilon c_{x} \hat{\tau}_{x}+\tilde{\sigma}_{x x}\right),
$$$$
0=\epsilon \frac{\partial \tilde{v}}{\partial \tilde{y}}-\tilde{A} \tilde{F}\left(2 \epsilon c_{y} \hat{\tau}_{y}+\tilde{\sigma}_{y y}\right),
$$

$$
0=\epsilon\left(\frac{\partial \tilde{u}}{\partial \tilde{y}}+\frac{\partial \tilde{v}}{\partial \tilde{x}}\right)-2 \tilde{A} \tilde{F}\left(\tilde{\tau}_{x y}+\epsilon c_{y} \hat{\tau}_{x}+\epsilon c_{x} \hat{\tau}_{y}\right)
$$

with the newly introduced variables

$$
\begin{aligned}
& \hat{\tau}_{x}=\tilde{\tau}_{x z}-\epsilon\left(c_{y} \tilde{\tau}_{x y}+c_{x} \tilde{\sigma}_{y y}+2 c_{x} \tilde{\sigma}_{x x}\right), \\
& \hat{\tau}_{y}=\tilde{\tau}_{y z}-\epsilon\left(c_{x} \tilde{\tau}_{x y}+c_{y} \tilde{\sigma}_{x x}+2 c_{y} \tilde{\sigma}_{y y}\right)
\end{aligned}
$$

and the abbreviations

$$
\begin{aligned}
& c_{x}=(1-\zeta) \frac{\partial \tilde{B}}{\partial \tilde{x}}+\zeta \frac{\partial \tilde{S}}{\partial \tilde{x}}, \\
& c_{y}=(1-\zeta) \frac{\partial \tilde{B}}{\partial \tilde{y}}+\zeta \frac{\partial \tilde{S}}{\partial \tilde{y}} .
\end{aligned}
$$

The quantities $c_{x}$ and $c_{y}$ correspond to the inclinations in the $\tilde{x}$ and $\tilde{y}$ directions of the surface $\zeta=$ const. through the given point $P(\tilde{x}, \tilde{y})$ in the ice. Introduction of new variables $\hat{\tau}_{x}$ and $\hat{\tau}_{y}$ transforms Equations (3.2) and (3.3) to a form that can be integrated using the method of lines. Additionally, $\hat{\tau}_{x}$ and $\hat{\tau}_{y}$ have a simple physical interpretation. To first-order approximation, they correspond to the components of the shear traction with respect to the surface $\zeta=$ const. Consequently, the first-order boundary conditions (Equations (2.39) and (2.40)) at the free upper surface $S$, which corresponds to the surface $\zeta=1$, yield

$$
\hat{\tau}_{x, \mathrm{~s}}=\hat{\tau}_{y, \mathrm{~s}}=0 .
$$

\subsection{Integration}

Introducing a discrete grid (Fig. 1) and approximating the $\tilde{x}$ and $\tilde{y}$ derivatives by centred differences, Equations (3.2)-(3.6) can be rewritten as ordinary differential equations (ODE) and Equations (3.7)-(3.9) become algebraic. For each vertical grid line $(i, j)$, this establishes a set of five ordinary differential equations for the five unknowns $\hat{\tau}_{x, i j}, \hat{\tau}_{y, i j}, \tilde{u}_{i j}, \tilde{v}_{i j}$ and $\tilde{w}_{i j}$, and three coupled algebraic equations for $\tilde{\sigma}_{x x, i j}, \tilde{\sigma}_{y y, i j}$ and $\tilde{\tau}_{x y, i j}$. These equations can be integrated simultaneously starting at the base, by using an ODE solver and solving the three algebraic equations by using an efficient root finder. To test the algorithm, second-order centred differences were used for the $\tilde{x}$ and $\tilde{y}$ derivatives, a Runge-Kutta scheme of second-order for integrating the ODEs, and a Newton Raphson algorithm for solving the algebraic equations.

This integration, started at $\zeta=0$ with some prescribed basal values for shear stresses and velocity components, does not automatically satisfy the surface boundary conditions in Equation (3.14). In order to solve the boundary-value problem, the proper basal values for $\hat{\tau}_{x, i j, \mathrm{~b}}, \hat{\tau}_{y, i j \mathrm{~b}, \mathrm{~b}}, \tilde{u}_{i j, \mathrm{~b}}, \tilde{v}_{i j, \mathrm{~b}}$ and $\tilde{w}_{i j, \mathrm{~b}}$ must be found iteratively. For non-sliding conditions, we have $\tilde{w}_{i j, \mathrm{~b}}=\tilde{u}_{i j, \mathrm{~b}}=\tilde{v}_{i j, \mathrm{~b}}=0$ and for sliding conditions we need the information for basal sliding velocities from some sort of sliding law. In both cases, basal velocities are given and we need to find $\hat{\tau}_{x, i j, \mathrm{~b}}$ and $\hat{\tau}_{y, i j, \mathrm{~b}}$. A good initial choice is

$$
\begin{aligned}
& \hat{\tau}_{x, i j, \mathrm{~b}}^{0}=-\left(\tilde{S}_{i j}-\tilde{B}_{i j}\right)\left(\frac{\partial \tilde{S}}{\partial \tilde{x}}\right)_{i j}, \\
& \hat{\tau}_{y, i j, \mathrm{~b}}^{0}=-\left(\tilde{S}_{i j}-\tilde{B}_{i j}\right)\left(\frac{\partial \tilde{S}}{\partial \tilde{y}}\right)_{i j},
\end{aligned}
$$

which corresponds to the shallow-ice approximation of the basal shear stresses. With these assumptions, the algebraic Equations (3.7)-(3.9) can be solved for the basal values of $\tilde{\sigma}_{x x, i j, \mathrm{~b}}, \tilde{\sigma}_{y y, i j, \mathrm{~b}}, \tilde{\tau}_{x y, i j, \mathrm{~b}}$. The subsequent integration from the base to the surface yields values

$$
\hat{\tau}_{x, i j, \mathrm{~s}}^{0} \neq 0 \text { and } \hat{\tau}_{y, i j, \mathrm{~s}}^{0} \neq 0,
$$

which generally do not meet the boundary conditions in Equation (3.14). The iteration is carried out by subsequently choosing new values

$$
\hat{\tau}_{x, i j, \mathrm{~b}}^{k+1}=\hat{\tau}_{x, i j, \mathrm{~b}}^{k}-\beta_{x} \hat{\tau}_{x, i j, \mathrm{~s}}^{k},
$$




$$
\hat{\tau}_{y, i j, \mathrm{~b}}^{k+1}=\hat{\tau}_{y, i j, \mathrm{~b}}^{k}-\beta_{y} \hat{\tau}_{y, i j, \mathrm{~s}}^{k}
$$

where $\beta_{x}$ and $\beta_{y}$ are chosen iteration parameters.

It is interesting to note that the solution for $\tilde{w}$ of the continuity Equation (3.4) does not feed back to the solutions of the seven remaining equations, and can be uncoupled from the iteration scheme. In fact, the continuity equation in any form can then be reduced to a quadrature, e.g. Equation (3.4) yields

$$
\tilde{w}=\tilde{w}_{\mathrm{b}}-\frac{1}{\epsilon}(\tilde{S}-\tilde{B}) \int_{0}^{\zeta} \tilde{A} \tilde{F}\left(\tilde{\sigma}_{x x}+\tilde{\sigma}_{y y}\right) d \zeta^{\prime},
$$

and can be integrated separately after the iteration has converged. In the rest of this paper, the scaled Equations (3.2)-(3.9) are used. The unscaled version of these equations can be recovered by setting $\epsilon=1$ and using the unscaled variables instead of the scaled ones.

\subsection{Plane-strain approximation}

Let us assume that no quantity depends on $\tilde{y}$, then $\partial / \partial \tilde{y} \equiv 0$. From Equation (2.26), it then follows that $\tilde{\sigma}_{y y} \equiv 0$. If we further assume that ice flows parallel to the $\tilde{x} \zeta$ plane, $\tilde{v} \equiv 0$, then from Equations (2.28) and (2.29) it follows that $\tilde{\tau}_{x y}=\tilde{\tau}_{y z} \equiv 0$; however $\tilde{\tau}_{y y} \neq 0$. With these assumptions, the set of eight equations, Equations (3.2)(3.9), reduces to

$$
\begin{gathered}
\frac{\partial \hat{\tau}}{\partial \zeta}=-2 \epsilon \frac{\partial c}{\partial \zeta} \tilde{\sigma}_{x x}-(\tilde{S}-\tilde{B})\left(2 \epsilon \frac{\partial \tilde{\sigma}_{x x}}{\partial \tilde{x}}-\frac{\partial \tilde{S}}{\partial \tilde{x}}\right), \\
\frac{\partial \tilde{w}}{\partial \zeta}=-\frac{1}{\epsilon}(\tilde{S}-\tilde{B}) \tilde{A} \tilde{F}(\cdot) \tilde{\sigma}_{x x}, \\
\frac{\partial \tilde{u}}{\partial \zeta}=2(\tilde{S}-\tilde{B}) \tilde{A} \tilde{F}(\cdot)\left(\hat{\tau}+2 \epsilon c \tilde{\sigma}_{x x}\right), \\
0=\epsilon \frac{\partial \tilde{u}}{\partial \tilde{x}}-\tilde{A} \tilde{F}(\cdot)\left(2 \epsilon c \hat{\tau}-\tilde{\sigma}_{x x}\right)
\end{gathered}
$$

with the new variable

$$
\hat{\tau}=\tilde{\tau}_{x z}-2 \epsilon c \tilde{\sigma}_{x x}
$$

and the abbreviation

$$
c=(1-\zeta) \frac{\partial \tilde{B}}{\partial \tilde{x}}+\zeta \frac{\partial \tilde{S}}{\partial \tilde{x}} .
$$

The transformed boundary condition for stress at the free surface is

$$
\hat{\tau}_{\mathrm{s}}=0 \text {, }
$$

The iteration scheme is the same as for the threedimensional case. An initial choice is

$$
\hat{\tau}_{i, \mathrm{~b}}^{0}=-\left(\tilde{S}_{i}-\tilde{B}_{i}\right) \frac{\partial \tilde{S}}{\partial \tilde{x}}
$$

which corresponds to the shallow-ice approximation of the basal shear stress. With this shear stress and the prescribed values for the basal-velocity components, Equation (3.24) is used to calculate the basal value for $\tilde{\sigma}_{x x, i, \mathrm{~b}}$. Integrating up from the base yields surface values $\hat{\tau}_{i, \mathrm{~s}}^{0} \neq 0$. The iteration is carried out by subsequently choosing a new value

$$
\hat{\tau}_{i, \mathrm{~b}}^{k+1}=\hat{\tau}_{i, \mathrm{~b}}^{k}-\beta \hat{\tau}_{i, \mathrm{~s}}^{k}
$$

where $\beta$ is the iteration parameter.

\section{PERFORMANGE TESTING OF THE ALGORITHM}

\subsection{Stability and convergence pattern}

Muller (1991) provided a detailed analysis of the accuracy and stability of the two-dimensional algorithm for a Navier-Stokes fluid. He gave a criterion for the convergence of the iteration scheme

$$
0<\beta<2 \exp \left(-\frac{2 M\{H\}}{\{L\}}\right)=2 \exp \left(-\frac{2 \epsilon}{\Delta \tilde{x}}\right)
$$

where $M$ is the number of grid points in the horizontal direction, and $\{H\}$ and $\{L\}$ are the vertical and horizontal extents of the ice mass, respectively. Equation (4.1) suggests a strong dependence of the convergence range on the aspect ratio $\epsilon=\{H\} /\{L\}$ and the chosen longitudinal grid resolution $\Delta \tilde{x}=1 / M$. The criterion in Equation (4.1) uses global quantities of the ice geometry. However, local conditions may require a smaller $\beta$ to achieve convergence. This is especially true if the surface slope locally displays large longitudinal variations, such as in icefalls or near-steep glacier snouts. The iteration process must start with larger residuals when the aspect ratio is larger and, additionally, the iteration parameter $\beta$ must be chosen smaller for larger $\epsilon$, which also makes the convergence progress slower. Both effects together make the number of necessary iteration steps grow rapidly with increasing $\epsilon$. Furthermore, if the ratio $\epsilon / \Delta \tilde{x}$ is too large, convergence cannot be achieved at all, no matter how small the iteration parameter $\beta$ is chosen.

The aim of the iteration is to approach the surfaceboundary condition $\hat{\tau}=0$. The convergence rate was tracked in many numerical experiments by monitoring the largest residual $\Delta \hat{\tau}_{s}$, until this residual drops below a prescribed level. If the iteration parameter $\beta$ is chosen much too large, the residuals start growing right at the beginning of the iteration. With $\beta$ close to the convergence range, the iteration often starts promisingly with steadily decreasing residuals but, at a certain point, convergence becomes very slow or stops altogether. For an appropriate choice of $\beta$, the convergence progresses approximately exponentially until round-off errors stop it and the residual begins to vary randomly within the round-off error interval. For single-precision computation (eight relevant digits), the levelling-off occurs near the $1 \mathrm{~Pa}$ level, which is considered to be accurate enough for the convergence threshold. Figure 2 illustrates the various convergence/divergence patterns for the scaled planestrain geometry shown in Figure 3, with an aspect ratio 


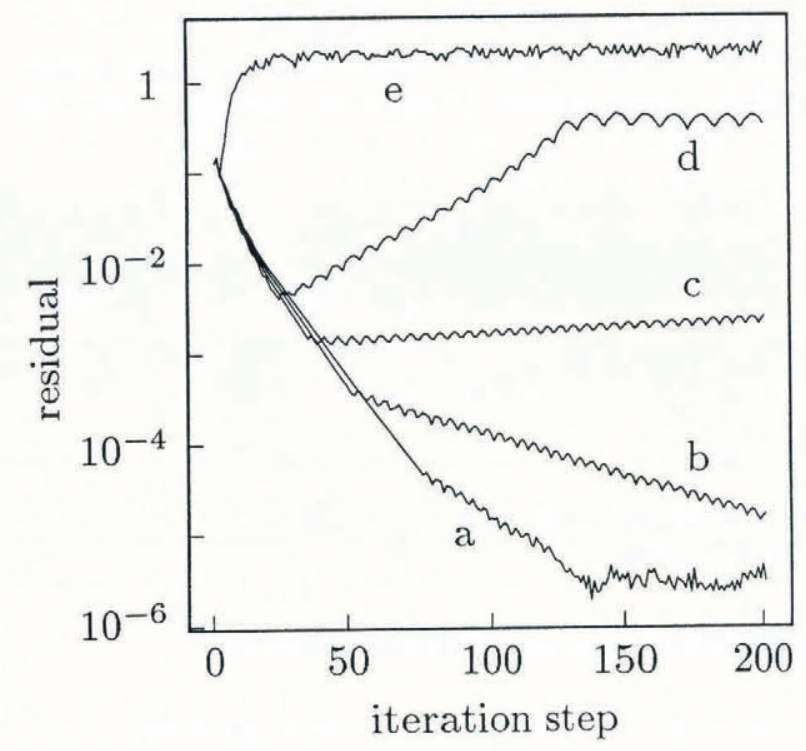

Fig. 2. Convergence/divergence patterns for various iteration parameters: (a) $\beta=0.065$, (b) 0.09, (c) 0.096 , (d) 0.105 and (e) 0.28 . The example corresponds to the scaled plane-strain cross-section as shown in Figure 2 and with an aspect ratio $\epsilon=0.05$. With the chosen grid resolution $\Delta \tilde{x}=0.025$, the convergence criterion (Equalion (3.31)) yields $\beta<0.037$.

$\epsilon=0.05$ and a horizontal grid resolution of $\Delta \tilde{x}=0.025$. Although the chosen geometry may not look very natural, the behaviour of the iteration is the same as for realistic glacier geometries.

\subsection{Scale length}

A given scaled geometry, $\tilde{S}=\tilde{S}(\tilde{x}, \tilde{y})$ and $\tilde{B}=\tilde{B}(\tilde{x}, \tilde{y})$ in the three-dimensional case or $\tilde{S}=\tilde{S}(\tilde{x})$, and $\tilde{B}=\tilde{B}(\tilde{x})$ in the plane-strain approximation, represents an infinite set of affine glacier geometries. For the case of a grounded ice mass with no basal sliding, the scaled solutions only depend on the temperature distribution, represented by $\tilde{A}=\tilde{A}(\tilde{x}, \tilde{y}, \tilde{z})$ or $\tilde{A}=\tilde{A}(\tilde{x}, \tilde{z})$, respectively, and the aspect ratio $\epsilon$. For isothermal ice masses, $\tilde{A} \equiv A_{0}$, with given geometry $\tilde{S}$ and $\tilde{B}$, the first-order equations only

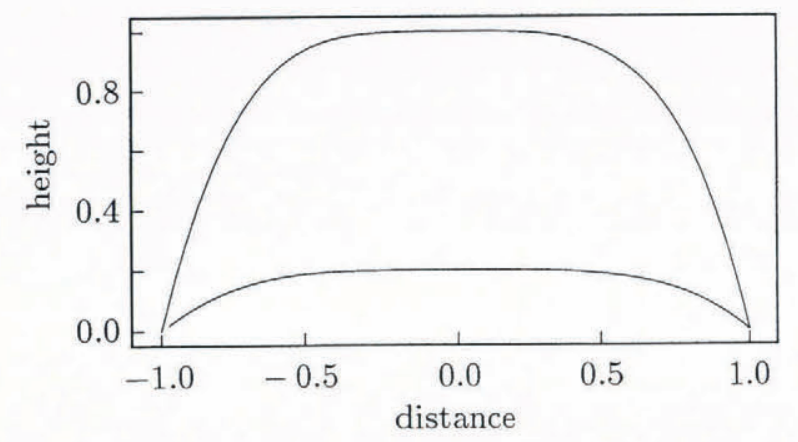

Fig. 3. Scaled cross-section, fourth-order parabola $\tilde{S}=$ $1-\tilde{x}^{4}$ and $\tilde{B}=0.2 \tilde{S}$, used for the numerical experiments, of which the results are illustrated in Figures $4-8$. The central thickness of the ice mass is $0.8\{\mathrm{H}\}$ and its diameter is $2\{L\}$. depend on one single parameter, the aspect ratio $\epsilon$. The shallow-ice approximation becomes unique by setting $\epsilon=0$ in Equations (3.21)-(3.24) or in Equations (3.2)(3.9). It is interesting to note that, in this case, the stress fields do not depend on the choice of $A_{0}$ but the velocity fields are proportional to $A_{0}$.

It seems reasonable to assume that the largest basal shear traction occurring in ice sheets and glaciers of a wide variety of shapes and sizes does not appreciably exceed $10^{5} \mathrm{~Pa}$. It is informative to reduce the set of shallow-ice masses with a given scaled shape $\tilde{S}$ and $\tilde{B}$ by fixing the maximum occurring basal shallow-ice shear stress at a given value $\tau_{0}$. The true size of an ice mass is now a function of $\epsilon$ and $\tau_{0}$. In this case, the scaled shallow-ice approximation is unique and thus does not depend on $\epsilon$ and $\tau_{0}$. From the scaling definition, Equations (2.17) and (2.19), it follows that

$$
\{L\} \propto\{H\}^{2} \propto \frac{1}{\epsilon^{2}}
$$

for the shallow ice approximation. Similarly, with Equation (2.18), it follows that $u \propto \epsilon^{-1}$, and $w$ is independent of $\epsilon$. Applying this result in Equation (3.24) yields

$$
\frac{w}{u} \propto \frac{\sigma_{x x}}{\tau_{x z}} \propto \epsilon,
$$

which indicates the growing importance of the normal deviatoric stress with growing aspect ratio $\epsilon$. A limiting basal shear traction of $10^{5} \mathrm{~Pa}$ now implies that even continent-size ice sheets cannot become thicker than a very few kilometres. On the other hand, this also implies the known fact that smaller ice masses tend to have larger aspect ratios, which makes the application of the described numerical algorithm more difficult; thus the iteration is slower for smaller glaciers than for larger ice caps and ice sheets.

This pattern is illustrated in the following numerical experiments using a simple synthetic geometry. As an example, a fourth-order parabola was chosen (Fig. 3) to represent the scaled images of the surface and basal profiles. These profiles were used in two ways. In the plane-strain approximation, they represent the geometry of a plane cross-section through an ice mass. In a second set of numerical experiments, these profiles are rotated around the $\bar{z}$ axis to define a circular ice sheet having cylindrical symmetry. Table 1 gives the scale values for $\{H\}$ and $\{L\}$ as a function of $\epsilon$ for this geometry and a maximum basal shallow-ice shear stress of $10^{5} \mathrm{~Pa}$.

Figure 4 shows the scaled values of the basal shear stress and the longitudinal deviatoric stress at the surface, and Figure 5 shows the velocity components at the surface for the plane-strain approximation. Figures 6 and 7 show the same quantities but for the radial section along the $\tilde{y}=0$ plane of the three-dimensional geometry. As can be expected, the shear stress and the radial velocity component in the shallow-ice approximation are the same for the plane-strain approximation and for the circular three-dimensional geometry. The differences between plane-strain and three-dimensional solutions of the first-order shear-stress and horizontal-velocity components are also small. However, the differences between plane-strain and the three-dimensional case are signif- 
Table 1. Scale values for $\{H\}=11.694 / \epsilon$ and $\{L\}=\{H\} / \epsilon$ for given $\epsilon$ for a plane-strain ice mass with scaled shape as shown in Figure 3, if the maximum occurring basal shear stress is $10^{5} \mathrm{~Pa}$. The smallest possible number of iteration steps, $n$, the corresponding best choice for the iteration parameter $\beta$, and the largest residual $\Delta \hat{\tau}_{0}$ after the first iteration step are given for some examples of $\epsilon$ and $\Delta \tilde{x}$. The theoretical upper limil for the iteration parameter $\beta_{\text {theor }}($ Equation (4.1)) is given for comparison. The iteration was slarted with the shallow-ice basal shear stress and the convergence threshold was chosen al $10 \mathrm{~Pa}$

\begin{tabular}{|c|c|c|c|c|c|c|c|}
\hline $\begin{array}{l}\epsilon \\
\{H\}(\mathrm{m}) \\
\{L\}(\mathrm{km})\end{array}$ & $\begin{array}{l}0.05 \\
234 \\
4.7\end{array}$ & & & & & $\begin{array}{l}0 . \\
23 \\
46\end{array}$ & \\
\hline$\Delta \tilde{x}$ & 0.025 & 0.025 & 0.0125 & 0.025 & 0.0125 & 0.025 & 0.0125 \\
\hline$\Delta \hat{\tau}_{0}$ & 0.1297 & 0.0411 & 0.0487 & 0.0084 & 0.0105 & 0.0024 & 0.0032 \\
\hline$\beta_{\text {theor }}$ & 0.037 & 0.27 & 0.037 & 0.9 & 0.4 & 1.34 & 0.9 \\
\hline$\beta$ & 0.085 & 0.38 & 0.065 & 1.0 & 0.7 & 1.0 & 0.98 \\
\hline$n$ & 69 & 13 & 81 & 5 & 6 & 3 & 3 \\
\hline
\end{tabular}

icant for the radial deviatoric stress and the verticalvelocity component, which is mainly due to the spreading effect of the circular ice mass. Furthermore, deviatoric stress components and the vertical-velocity component strongly depend on the aspect ratio.

The cylindrical symmetry of the assumed threedimensional geometry should be reflected in the results for stress and velocity components. This is used to test the influence of grid size and the resulting polygonal margin of the circular ice mass. The solutions for the verticalvelocity component $w$ and the total horizontal velocity $v_{h}^{2}=u^{2}+v^{2}$ must be cylindrical. It is interesting to note that the same is true for the quadratic forms $\tau_{r z}{ }^{2}=$

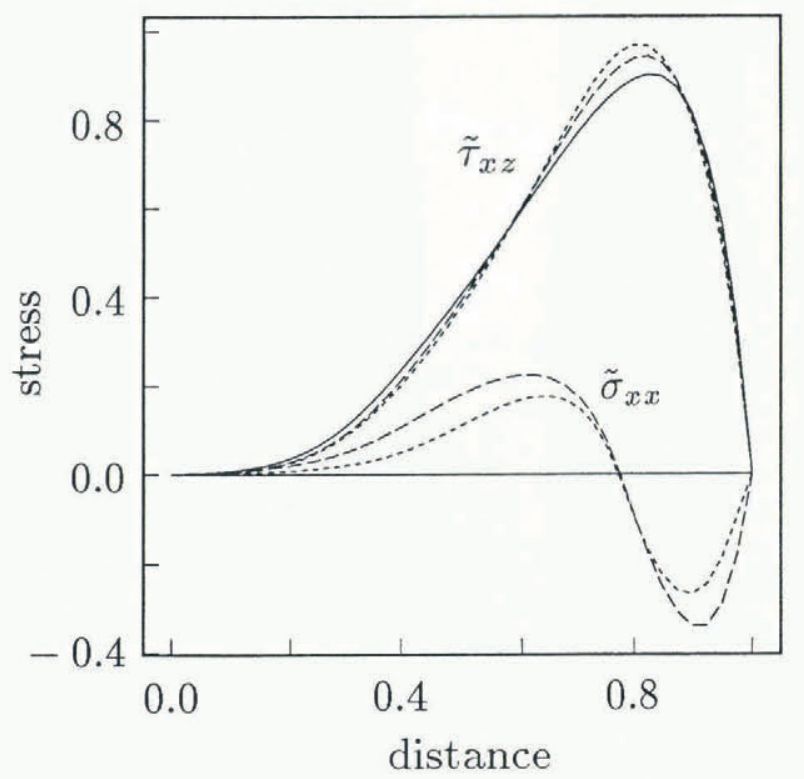

Fig. 4. Basal shear stress $\tilde{\tau}_{x z}$ and devialoric stress $\tilde{\sigma}_{x x}$ at the surface of the two-dimensional plane-strain section of the ice mass illustrated in Figure 2, for different aspect ratios: $\epsilon=0.05$ (solid lines), $\epsilon=0.025$ (dashed lines) and $\epsilon=0$ (dotted lines), and $\tilde{t}_{0}{ }^{2}=0.1$. The horizontal coordinate axis denotes the scaled distance from the centre of the ice mass. $\tau_{x z}{ }^{2}+\tau_{y z}{ }^{2}$ and $\sigma_{r r}{ }^{2}=\sigma_{y y}{ }^{2}+\sigma_{x x}{ }^{2}+\sigma_{x x} \sigma_{y y}+\tau_{x y}{ }^{2}$. It is straightforward to show that the quantity $\tau_{r z}$ is indeed an invariant under rotations of the coordinate system around the $z$ axis and the same holds for $\sigma_{r r}$, since the sum $\tau_{r z}{ }^{2}+\sigma_{r r}{ }^{2}$ equals the square of the effective stress, i.e. the second invariant of the deviatoric stress tensor. This invariance is not a result of the cylindrical symmetry of the chosen ice mass but is generally valid. Figure 8 shows a contour plot of the basal $\tau_{r z}$ for the circular ice sheet with cross-section as shown in Figure 3. The contours are close to circles except for a narrow marginal zone, where they seem to be influenced slightly by the polygonal shape of the margin.

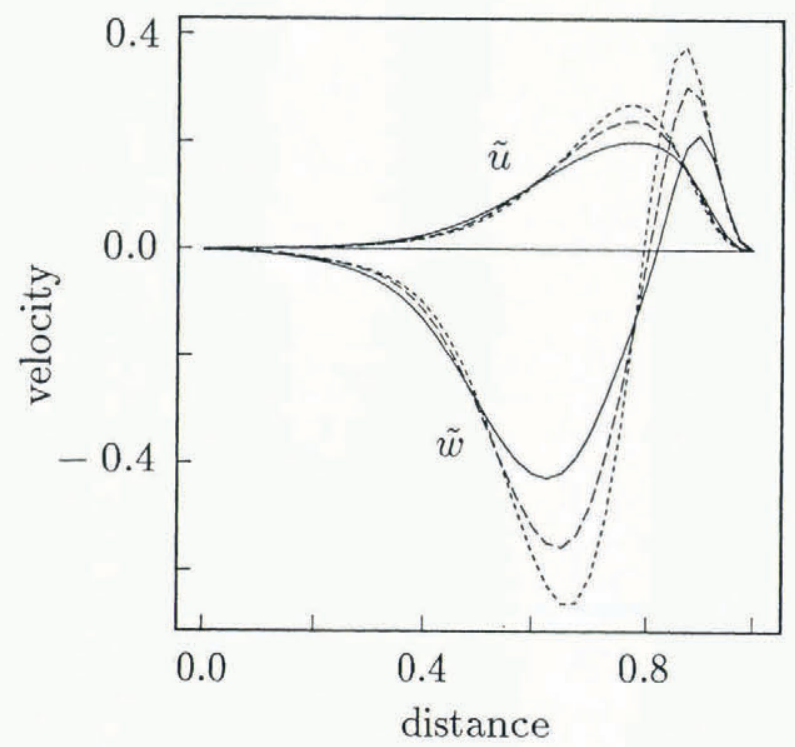

Fig. 5. Horizontal velocity component $\tilde{u}$ and vertical velocity component $\tilde{w}$ at the surface of the two-dimensional plane-strain section of the ice mass illustrated in Figure 2, for different aspect ratios: $\epsilon=0.05$ (solid lines), $\epsilon=0.025$ (dashed lines) and $\epsilon=0$ (dotted lines), and $\tilde{t}_{0}{ }^{2}=0.1$. The horizontal coordinate axis denotes the scaled distance from the centre of the ice mass. 


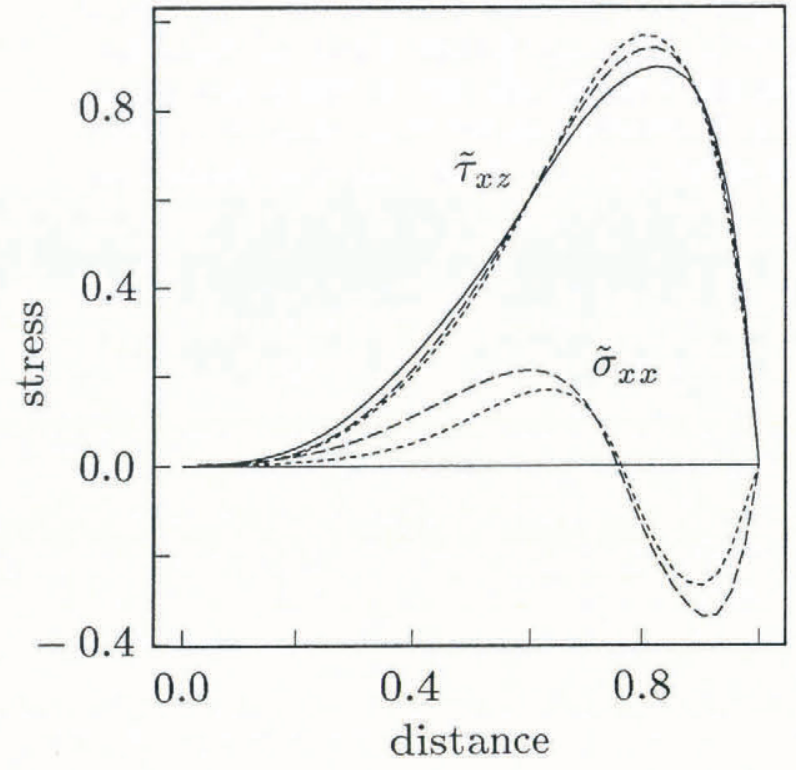

Fig. 6. Same as Figure 4 but in the radial cross-section along the plane $\tilde{y}=0$ of the three-dimensional circular ice mass with the cross-section illustrated in Figure 2.

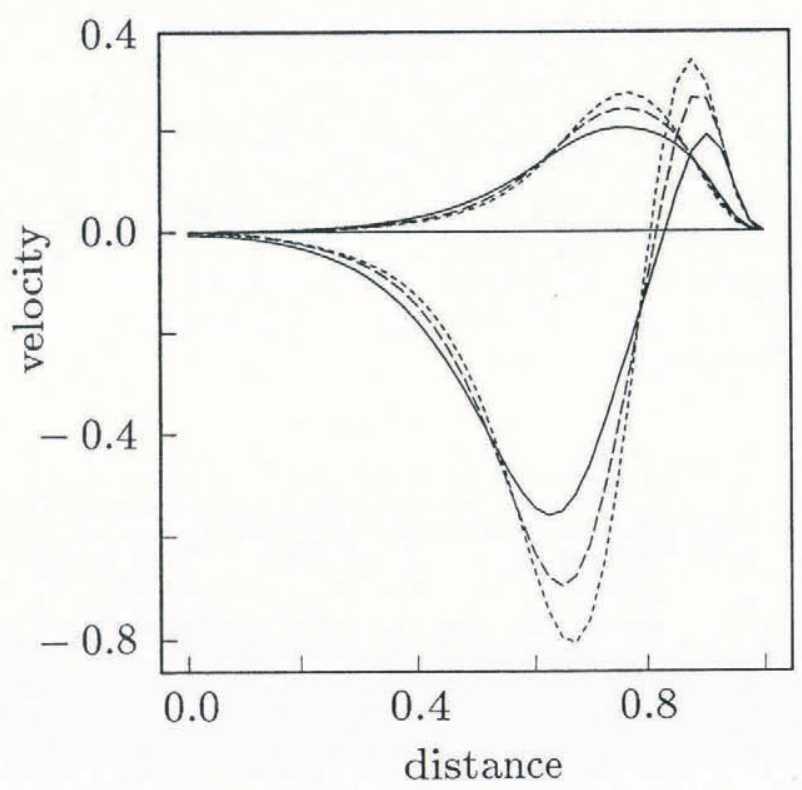

Fig. 7. Same as Figure 5 but in the radial cross-section along the plane $\bar{y}=0$ of the three-dimensional circular ice mass with the cross-section illustrated in Figure 2.

\subsection{Plane-strain parallel-sided slab}

In this section, the effect of longitudinal strain on the stress components is re-investigated Collins, 1968; Nye, 1969; Budd, 1970; Hutter, 1981) by applying the firstorder algorithm to a plane-strain parallel-sided slab geometry. This simple geometry is chosen to separate the effect of longitudinal strain from effects due to changes in ice thickness or longitudinal variations in the ice temperature. The coordinate system $(x, z)$ is chosen to lie parallel to the slab direction with the $z$ axis pointing perpendicular to the slab. The momentum equations are

$$
\begin{aligned}
& \frac{\partial \tau_{x x}}{\partial x}+\frac{\partial \tau_{x z}}{\partial z}=-\rho g \sin \alpha, \\
& \frac{\partial \tau_{x z}}{\partial x}+\frac{\partial \tau_{z z}}{\partial z}=\rho g \cos \alpha
\end{aligned}
$$

where $\alpha$ is the inclination angle of the slab normal with respect to the direction of gravity. The same procedure as described in section 2.2 yields the corresponding firstorder stress equation

$$
2 \frac{\partial \sigma_{x x}}{\partial x}+\frac{\partial \tau_{x z}}{\partial z}=\rho g\left(\frac{\partial S}{\partial x} \cos \alpha-\sin \alpha\right) .
$$

For the parallel-sided slab with surface $S \equiv H$ and base $B \equiv 0$, we get $\partial S / \partial x \equiv 0$.

The slab is defined by its thickness $H$ and its inclination angle $\alpha$, which suggests a somewhat different scaling than used in the previous sections:

$$
\left(\tau_{x z}, \sigma_{x x}\right)=\rho g H \sin \alpha(\tilde{\tau}, \tilde{\sigma}),
$$

$$
(u, w)=A_{0} H(\rho g H \sin \alpha)^{n+1}(\tilde{u}, \tilde{w}),
$$

$$
(x, z)=H(\tilde{x}, \tilde{z})
$$

where $A_{0}$ is a typical rate factor. For an isothermal slab, $A \equiv A_{0}$, Equations (4.6) and (3.22)-(3.24) in scaled form are

$$
\begin{aligned}
\frac{\partial \tilde{\tau}}{\partial \tilde{z}} & =-2 \frac{\partial \tilde{\sigma}}{\partial \tilde{x}}-1, \\
\frac{\partial \tilde{w}}{\partial \tilde{z}} & =-\tilde{F}(\cdot) \tilde{\sigma}, \\
\frac{\partial \tilde{u}}{\partial \tilde{z}} & =2 \tilde{F}(\cdot) \tilde{\tau}, \\
0 & =\frac{\partial \tilde{u}}{\partial \tilde{x}}-\tilde{F}(\cdot) \tilde{\sigma},
\end{aligned}
$$

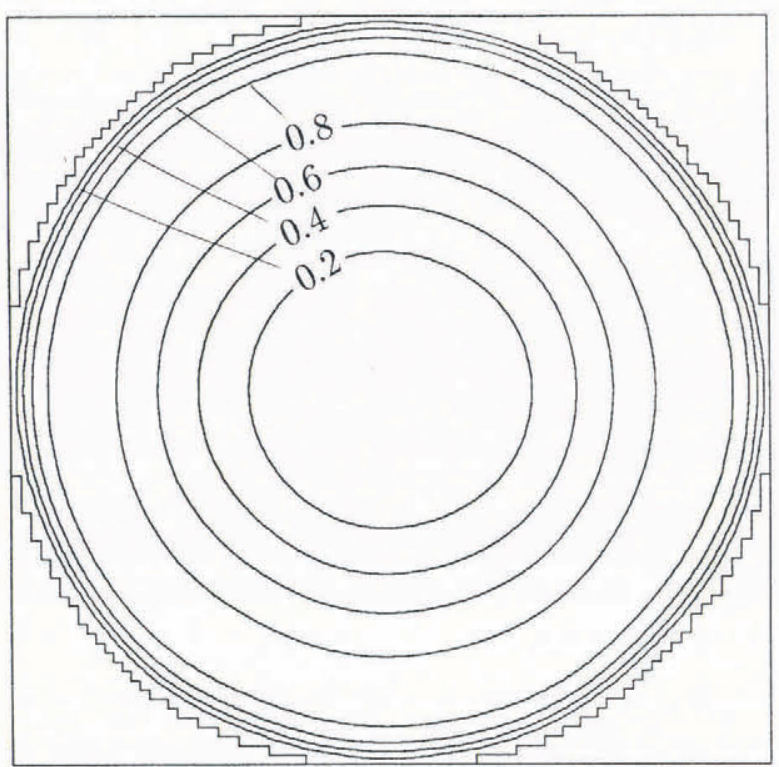

Fig. 8. Contour plot of $\tilde{\tau}_{r z}$, defined by $\tilde{\tau}_{r z}{ }^{2}=\tilde{\tau}_{x z}{ }^{2}+\tilde{\tau}_{y z}{ }^{2}$, at the base of the circular ice mass with the cross-section illustrated in Figure 2. The aspect ratio is $\epsilon=0.0 .5$ and $\tilde{\tau}_{0}{ }^{2}=0.1$. The polygonal line represents the discretized margin of the circular ice sheet. 
again with the flow law of Equation (2.10). For the slab geometry, the scaling alone yields a set of equations suitable for the first-order algorithm and the only remaining parameter is $\tilde{t}_{0}^{2}$ in the flow law $\tilde{F}(\cdot)$.

For a parallel sided slab, the characteristic longitudinal length scale $\{L\}$, and thus the aspect ratio $\epsilon$, can be defined by $H /\{L\}=\epsilon=\sin \alpha$. With this, the stability criterion in Equation (4.1) becomes

$$
0<\beta<2 \exp \left(-\frac{2}{\Delta \tilde{x}}\right) .
$$

The stability of the iteration does not depend on the slab inclination, as also can be concluded from the scaled Equations (4.10)-(4.13), which no longer contain the angle $\alpha$. Moreover, convergence could only be reached for $\Delta \tilde{x} \geq 1$, which limits the resolution of the longitudinal grid spacing to roughly one slab thickness. For Glen's flow law, with $\tilde{t}_{0}{ }^{2}=0$, the iteration sometimes stalled, possibly due to the singularity occurring at positions where stress vanishes, e.g. near the ice surface in the locations where longitudinal strain vanishes. This is a problematic feature of the Glen flow law and confirms the necessity of knowing the flow law accurately, especially if effects of longitudinal strain are taken into account.

\subsection{Effect of longitudinal strain}

In the shallow-ice approximation, the shear stress is independent of longitudinal strain. This is no longer true for the first-order approximation. A look at the first-order equations for a Navier-Stokes fluid

$$
\tilde{F}(\cdot)=\tilde{t}_{0}^{2}
$$

reveals an interesting relation between longitudinal velocity variations and shear stress. With Equation (4.15), the algebraic Equation (4.13) becomes linear in $\tilde{\sigma}$ and can be solved for $\tilde{\sigma}$. Applying the solution in Equation (4.10) gives

$$
\frac{\partial \tilde{\tau}}{\partial \tilde{z}}=-\frac{2}{\tilde{t}_{0}^{2}} \frac{\partial^{2} \tilde{u}}{\partial \tilde{x}^{2}}-1 .
$$

To avoid a singularity in the shear stress, $\partial^{2} \tilde{u} / \partial \tilde{x}^{2}$ must be finite. This not only excludes a jump in the velocity field, which is ruled out for obvious reasons, but also a jump in the longitudinal gradient of the velocity field, which is less obvious. This may explain the singular results reported by Hutter and Olunloyo (1980) and Barcilon and MacAyeal (1993) for abrupt sliding/nonsliding transitions at the bed of ice slabs. With the flow law in Equation (2.10), the solutions for $\tilde{\sigma}$ of the firstorder Equation (4.13) also depend on $\partial \tilde{u} / \partial \tilde{x}$, and consequently the shear stress in Equation (4.10) depends on $\partial^{2} \tilde{u} / \partial \tilde{x}^{2}$. Thus, to avoid a singularity in the stress field, the same conditions as for a Navier Stokes fluid must be met.

On the other hand, for a Navier-Stokes fluid, the shear stress only deviates from the shallow-ice shear stress if the longitudinal gradient of the velocity field also changes with $\tilde{x}$, i.e. $\partial^{2} \tilde{u} / \partial \tilde{x}^{2} \neq 0$. The same pattern holds for a generalized Glen-type fluid. Equations (4.10)-(4.13) contain only first derivatives with respect to $\tilde{x}$ and $\tilde{z}$ of the velocity components $\tilde{u}$ and $\tilde{w}$. Therefore, if $(\tilde{u}, \tilde{w})$ is a solution of the equations, then $\left(\tilde{u}+\tilde{u}_{0}, \tilde{w}\right)$ is also a solution, meeting the boundary conditions for stress and $\tilde{w}_{\mathrm{b}}=0$. The constant field $\tilde{u}_{0}$ plays a role equivalent to a global change in the sliding velocity. When the sliding velocity is a linear function of $\tilde{x}$, or equivalently

$$
\frac{\partial \tilde{u}_{\mathrm{b}}}{\partial \tilde{x}}=\text { const., }
$$

a change in $\tilde{u}_{0}$ is also equivalent to a shift of the velocity field parallel to the $\tilde{x}$ axis, hence

$$
\begin{gathered}
\frac{\partial \tilde{u}}{\partial \tilde{x}} \equiv \frac{\partial \tilde{u}_{\mathrm{b}}}{\partial \tilde{x}} \equiv \text { const., } \\
\frac{\partial^{2} \tilde{u}}{\partial \tilde{x}^{2}} \equiv \frac{\partial \tilde{w}}{\partial \tilde{x}} \equiv \frac{\partial \tilde{\sigma}}{\partial \tilde{x}} \equiv \frac{\partial \tilde{\tau}}{\partial \tilde{x}} \equiv 0 .
\end{gathered}
$$

To study the effect of longitudinal strain on the stress field, the shear stress and the longitudinal deviatoric stresses were calculated as a function of the prescribed longitudinal variations of the basal-velocity field. The algorithm was used to solve Equations $(4.10)-(4.13)$ for a non-linear flow law (Equation (2.10)) and $n=3$. Vertical profiles of stress and velocity components were prescribed as boundary conditions at the upper and lower ends of the slab with finite length. Near these ends, zones with no longitudinal strain were defined by prescribing constant basal-velocity components. Between these zones, the basal-velocity components were varied as required for the specific study.

A constant longitudinal gradient of the sliding velocity, Equation (4.17), was assumed in a first set of numerical experiments. A typical result is illustrated in Figure 9. The longitudinal stress at the surface of the slab remains zero in the homogeneous zones and is constant in the zone where sliding velocity increases linearly. The shear stress only varies slightly in the two positions where the longitudinal gradient of the sliding velocity changes but is unaffected in places where the longitudinal sliding gradient is constant. This confirms the pattern suggested by Equation (4.16) for a Navier-Stokes fluid, in so far as shear stress is undisturbed in this case and Equations (4.18) and (4.19) still hold. The dependence of the longitudinal stress on the longitudinal sliding gradient is depicted in Figure 10.

The shear velocity, $\tilde{u}_{\mathrm{s}}-\tilde{u}_{\mathrm{b}}$, also depends on the longitudinal gradient of the basal velocity. This is a result of the non-linear flow law Equation (2.10)), which contains the effective stress and thus, the longitudinal deviatoric stress $\tilde{\sigma}$, which makes the ice softer with increasing $\tilde{\sigma}$. Figure 11 illustrates the dependence of the shear velocity on the longitudinal gradient of the basal velocity for three different $\tilde{t}_{0}$, together with one example of a constant viscosity (Navier-Stokes fluid), for which the shear velocity no longer depends on the sliding gradient.

This cannot hold anymore in the case of a longitudinally changing sliding gradient. In the following example, a sliding velocity is a quadratic function of $\tilde{x}$. Figure 12 shows a typical result for a constant value of $\partial^{2} \tilde{u}_{\mathrm{b}} / \partial \tilde{x}^{2}$. The result indicates that the longitudinal 

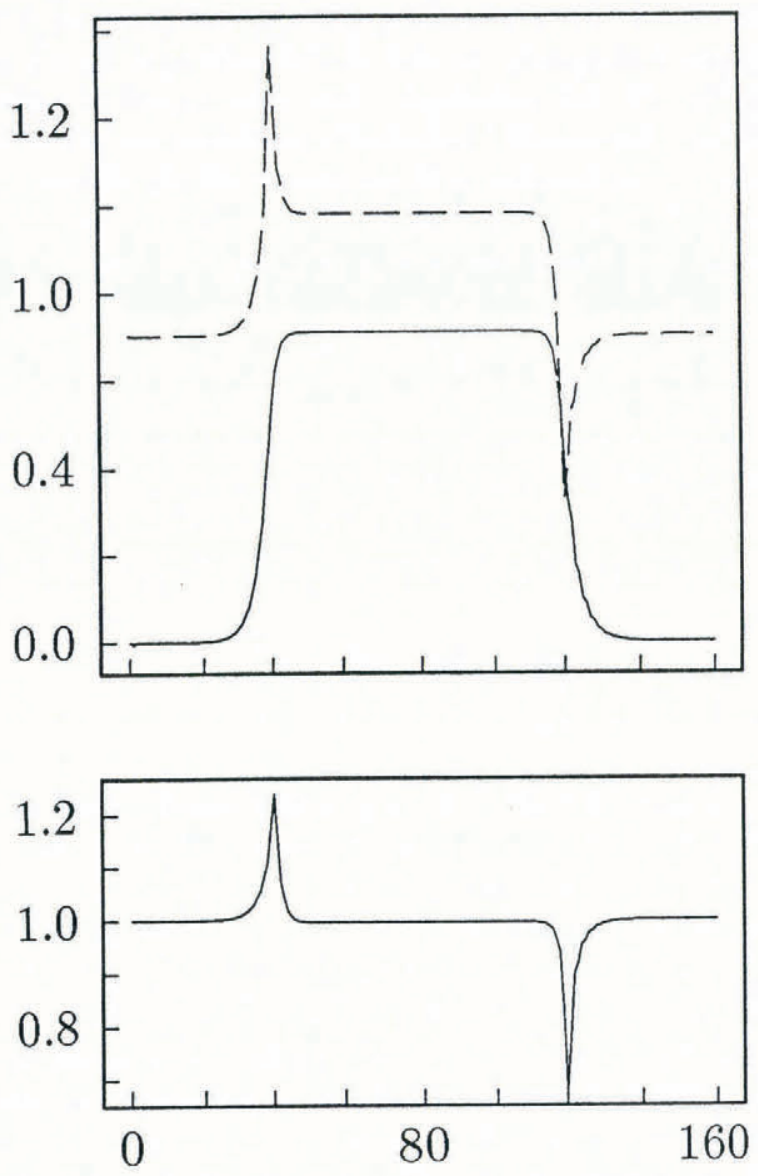

Fig. 9. Typical result for the dimensionless basal shear stress (solid line) and dimensionless longitudinal devialoric stress at the surface of the slab versus longitudinal spatial coordinate $\tilde{x}$, given by the number of the grid point. The chosen parameters are $\partial \tilde{u}_{\mathrm{b}} / \partial \tilde{x}=-0.5$ (stretching) and $\tilde{\tau}_{0}{ }^{2}=0.25$. The unit on the $\tilde{x}$ axis corresponds to one slab thickness.

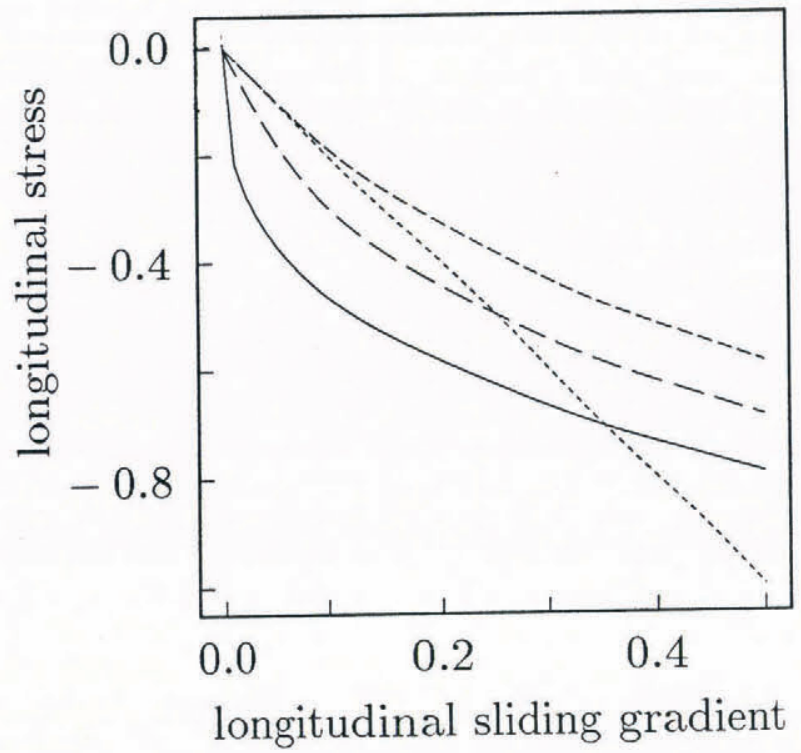

Fig. 10. Dimensionless longitudinal deviatoric stress at the surface of the slab versus dimensionless longitudinal sliding gradient $\partial \tilde{u}_{\mathrm{b}} / \partial \tilde{x}$ for $\tilde{\tau}_{0}{ }^{2}=0$ (solid line), $\tilde{\tau}_{0}^{2}=0.25$ (long-dashed line) and $\tilde{\tau}_{0}{ }^{2}=0.5$ (dashed line). The dotted line represents the result for the Navier-Stokes fluid for $\tilde{\tau}_{0}^{2}=0.5$.

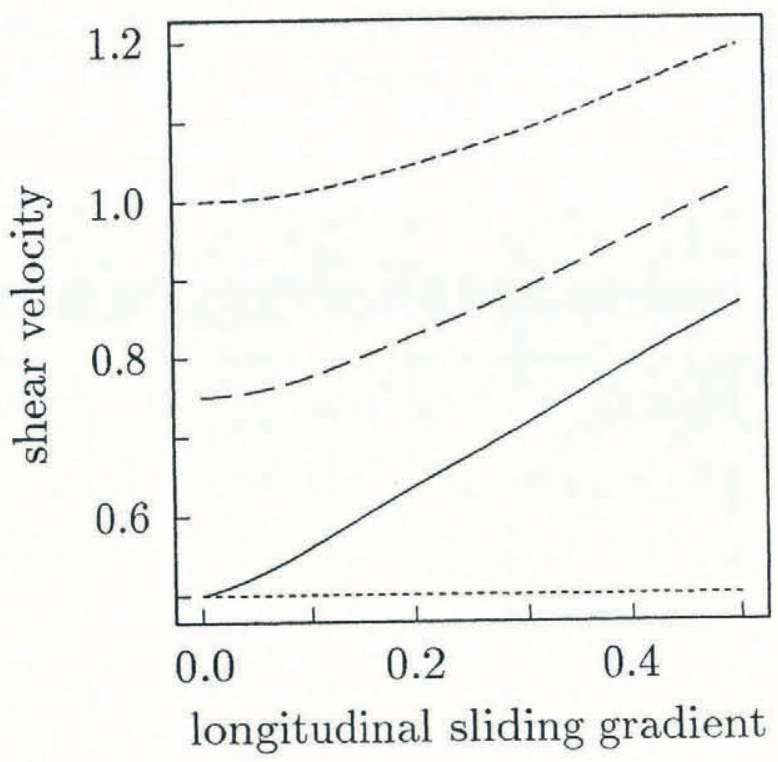

Fig. 11. Dimensionless shear velocity $\tilde{u}_{\mathrm{s}}-\tilde{u}_{\mathrm{b}}$ at the surface of the slab versus dimensionless longitudinal sliding gradient $\partial \tilde{u}_{\mathrm{b}} / \partial \tilde{x}$ for $\tilde{\tau}_{0}{ }^{2}=0$ (solid line), $\tilde{\tau}_{0}{ }^{2}=0.25$ (long-dashed line) and $\tilde{\tau}_{0}^{2}=0.5$ (dashed line). The dotted line represents the result for the Navier-Stokes fluid for $\tilde{\tau}_{0}^{2}=0.5$.

deviatoric stress is mostly determined by the local $\partial \tilde{u}_{\mathrm{b}} / \partial \tilde{x}$. Seemingly, the shear stress also depends mostly on the local variation of the sliding velocity: $\partial^{2} \tilde{u}_{\mathrm{b}} / \partial \tilde{x}^{2}$ and $\partial \tilde{u}_{\mathrm{b}} / \partial \tilde{x}$.

\section{CONCLUSIONS AND DISGUSSION}

The numerical algorithm developed by Muller (1991) for calculating stress and velocity fields in two-dimensional (plane-strain) grounded ice masses also takes into account the effects of the normal deviatoric stresses. The algorithm was extended to three dimensions and its applicability was examined for various situations. It works efficiently for large ice-sheet configurations and the necessary iteration to match the stress-boundary conditions at the surface converges rapidly. However, the efficiency decreases with increasing horizontal spatial resolution and with increasing aspect ratio, corresponding to a smaller ice mass. Compared with the isothermal case, the introduction of temperature layering also slows the iteration.

The algorithm can become unstable for an ice geometry having a large aspect ratio or a grid with too fine a longitudinal spatial step. Such instabilities often originate where horizontal gradients in surface or bed topography are large. Instabilities can arise in different parts of the algorithm: (1) the ODE integrator (e.g. Runge-Kutte scheme) may become unstable if the vertical grid size is inadequate, (2) the root finder (e.g. Newton-Raphson scheme) may become stalled or run away, and (3) the iteration procedure may diverge. The third cause is most frequent and limits the range of applicability of the algorithm. If convergence can be reached, the best choice for the iteration parameter to achieve fastest convergence must be found by experiment 

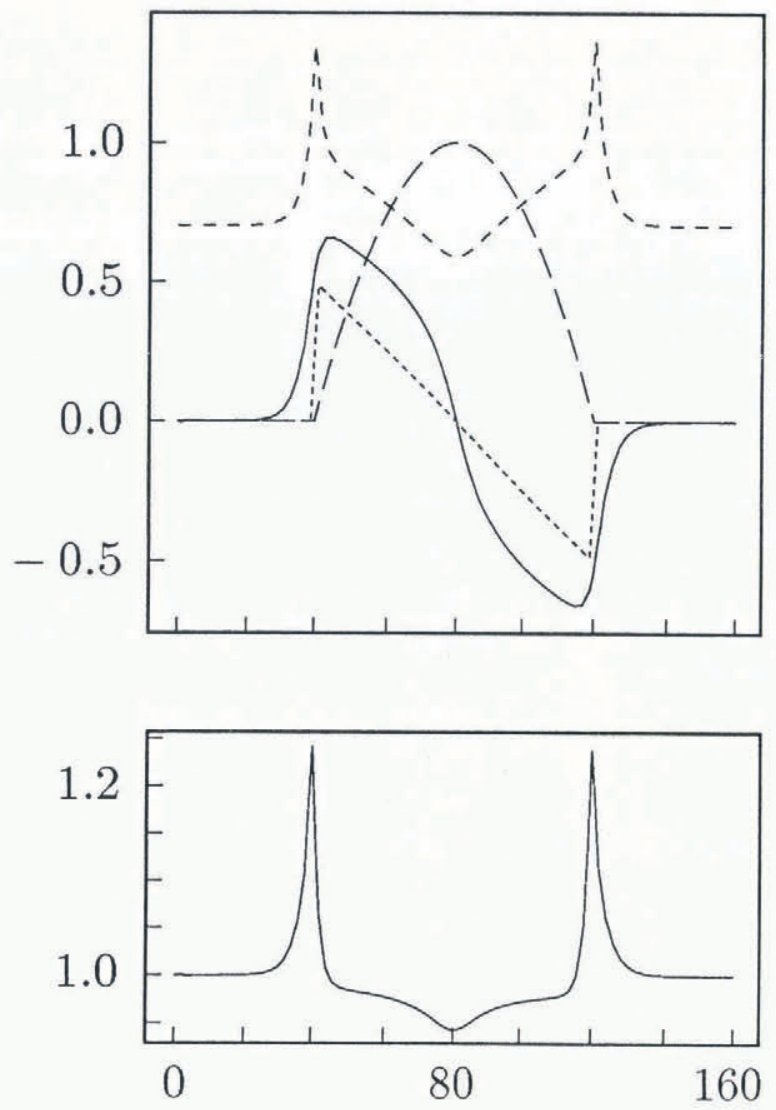

Fig. 12. Dimensionless basal shear stress (lower graph), longitudinal deviatoric stress at the surface of the slab (solid line in upper graph) and dimensionless sliding velocity $\tilde{u}_{\mathrm{b}} / 10$ (long-dashed line) versus longitudinal spatial coordinate $\tilde{x}$, given by the number of the grid point. The dashed line depicts the resulting shear velocity $\tilde{u}_{\mathrm{s}}-\tilde{u}_{\mathrm{b}}$ and the dolted line the longitudinal sliding gradient $\partial \tilde{u}_{\mathrm{b}} / \partial \tilde{x}$. In the sliding part of the slab, the second derivative of the sliding velocity $\partial^{2} \tilde{u}_{\mathrm{b}} / \partial \tilde{x}^{2}=$ 0.037 . The unit on the $\tilde{x}$ axis corresponds to one slab thickness.

for each geometry and aspect ratio.

The limits of applicability of the algorithm were explored and this points the direction for future research. The value of the method could be greatly enhanced if the horizontal resolution of the discretization grid could be improved. This is an essential prerequisite addressing such interesting questions as the stress field near a calving front, and the patchy basal stress and velocity field of sliding glaciers, where the characteristic size of the patchiness may be smaller than the average ice thickness (personal communication from G. K. C. Clarke).

The inclusion of floating ice shelves would necessitate a hybrid iteration procedure. In the ice-shelf part, the shear traction vanishes not only at the upper ice surface but at the floating base. To adjust to this situation, it would be necessary to specify the velocity at the floating base and shoot to satisfy the stress-boundary condition at the upper surface. As shown in section 4.4, the relation of the stress field to the basal velocity field is weak and more complex than the relation of the stress field to the basal shear stress. This makes the iteration difficult for the floating parts and, thus far, no converging iteration procedure has been found. Van der Veen (1987) introduced an iteration scheme usable for both grounded and floating parts of an ice mass. However, the applied vertical averaging of the longitudinal stress may be a viable option for floating ice shelves but may be difficult to justify for grounded glaciers.

Despite the above-mentioned limitations, the algorithm works efficiently for an interesting range of ice-sheet and glacier configurations. Its coding is very simple, which makes its application very flexible. The required input is the surface and basal geometry, and the basal velocity. In contrast to other numerical schemes incorporating deviatoric stress gradients, it is not necessary to start the iteration with an initial guess for the whole stress and velocity fields. The only necessary field input is the rate factor, or equivalently, the temperature field, which may be supplied from other sources. The required initial guess for the basal shear traction can easily be calculated if the shallow-ice approximation is used or it can be taken from the previous time-step if the code is incorporated into a transient ice-sheet model. The computation time for one iteration step is comparable to the computation time for the shallow-ice approximation of the velocity field. For ice caps and ice sheets of intermediate $(100 \mathrm{~km})$ to large size, the algorithm usually converges within less than ten steps. The number of iteration steps drops to one or two for each time-step, if the surface geometry is not changing fast and the result of the previous time-step is used as a starting point. Thus, the algorithm can be readily incorporated in a thermo-mechanically coupled transient ice-sheet model.

\section{ACKNOWLEDGEMENTS}

This work was done while on leave at the Department of Geophysics and Astronomy, University of British Columbia, Vancouver. The author wishes to thank G. K.C. Clarke, S. Marshall and U. Fischer for stimulating discussions. G. K. C. Clarke reviewed an earlier version of the paper and helped to improve it substantially. A. Ohmura generously made the sabbatical leave possible. G. Wanner, Department of Mathematics, University of Geneva, supported the development of the algorithm in his Institute.

\section{REFERENGES}

Barcilon, V. and D. R. MacAyeal. 1993. Steady flow of a viscous ice stream across a no-slip/free-slip transition at the bed. I. Glaciol., 39 (131), 167-185.

Budd, W. F. 1970. The longitudinal stress and strain-rate gradients in ice masses. 7. Glaciol., 9 (5.5), 19-27.

Collins, I. F. 1968. On the use of the equilibrium equations and flow law in relating the surface and bed topography of glaciers and ice sheets. J. Glaciol., $7(50), 199-204$.

Dahl-Jensen, D. 1989. Steady thermomechanical flow along twodimensional flow lines in large grounded ice sheets. J. Geophys, Res., 94 B8), 10,355-10,362.

Glen, J. W. 1958. The flow law of ice. International Association of Scientific Hydrology Publication 47 (Symposium at Chamonix 1958 - Physics of the Movement of the Ice), 171-183.

Herterich, K. 1987. On the flow within the transition zone between ice sheet and ice shelf. In Van der Veen, C.J. and J. Oerlemans, eds. 
Dynamics of the West Antarctic ice sheet. Dordrecht, etc., D. Reidel Publishing Co., $185-202$.

Herterich, K. 1988. A three-dimensional model of the Antarctic ice sheet. Ann. Glaciol., 11, 3235.

Hutter, K. 1983. Theoretical glaciology; material science of ice and the mechanics of glaciers and ice sheets. Dordrecht, etc., D. Reidel Publishing Co. Tokyo, Terra Scientific Publishing Co.

Hutter, K. and V.O.S. Olunloyo. 1980. On the distribution of stress and velocity in an ice strip, which is partly sliding over and partly adhering to its bed, by using a Newtonian viscous approximation. Proc. R. Soc. London, Ser. A, 373 1754), 385-403.

Huybrechts, P. 1992. The Antarctic ice sheet and environmental change: a three-dimensional modelling study. Ber. Polarforsch. 99.

Jenssen, D. 1977. A three-dimensional polar ice-sheet model. J. Glaciol., $1880), 373-389$.

Mahaffy, M.W. 1976. A three-dimensional numerical model of ice sheets: tests on the Barnes Ice Cap, Northwest Territories. 7. Geophys. Res., 81 6), 1059-1066.

Meier, M.F. 1958. Vertical profiles of velocity and the flow law of glacier ice. International Association of Scientific Hydrology Publication 47 (Symposium at Chamonix 1958 - Physics of the Movement of the Ice), $169-170$.
Morland, L. W. 1984. Thermo-mechanical balances of ice sheet flows. Geophys. Astrophys. Fluid Dyn., 29, 237-266.

Morland, L. W. and E, M. Shoemaker. 1982. Ice shelf balances. Cold Reg. Sci. Technol., 5 3), 235-251.

Muller, H.C. 1991. Une méthode iterative simple pour résoudre les équations de mouvement d'un glacier. Mémoire de Diplôme en Mathématique, Université de Genève.)

Nye, J.F. 1953. The flow law of ice from measurements in glacier tunnels, laboratory experiments and the Jungfraufirn borehole experiment. Proc. R. Soc. London, Ser. A, 219 (1139), 477-489.

Nyc, J.F. 1969. The effect of longitudinal stress on the shear stress at the base of an ice sheet. J. Glaciol., 8 53), 207-213,

Phillips, N.A. 1957. A coordinate system having some advantages for numerical forecasting. 7. Meleorol., 14 2), $184-185$.

Van der Veen, C.J. 1987. Longitudinal stresses and basal sliding: a comparative study. In Van der Veen, C. J. and J. Oerlemans, eds. Dynamics of the West Antarctic ice sheet. Dordrecht, etc., Kluwer Academic Publishers, 223-248.

Weertman, J. 1973. Creep of ice. In Whalley, E., S.J. Jones and L. W. Gold, eds. Physics and chemistry of ice. Ottawa, Royal Society of Canada, 320-337.

MS received 8 July 1994 and accepted in revised form 8 . November 1994 